\title{
Analysis of Stochastic Delay Predator-Prey System with Impulsive Toxicant Input in Polluted Environments
}

\author{
Meng Liu \\ School of Mathematical Science, Huaiyin Normal University, Huaian 223300, China \\ Correspondence should be addressed to Meng Liu; liumeng0557@sina.com
}

Received 21 June 2013; Revised 28 August 2013; Accepted 28 August 2013

Academic Editor: Massimiliano Ferrara

Copyright (C) 2013 Meng Liu. This is an open access article distributed under the Creative Commons Attribution License, which permits unrestricted use, distribution, and reproduction in any medium, provided the original work is properly cited.

A stochastic delay predator-prey model in a polluted environment with impulsive toxicant input is proposed and studied. The thresholds between stability in time average and extinction of each population are obtained. Some recent results are extended and improved greatly. Several simulation figures are introduced to support the conclusions.

\section{Introduction}

Environmental pollution by industries, agriculture, and other human activities is one of the most important socioecological problems in the world today. Due to toxins in the environment, lots of species have gone extinct, and many are on the verge of extinction. Thus, controlling the environmental pollution and the conservation of biodiversity are the major focus areas of all the countries around the world. This motivates scholars to study the effects of toxins on populations and to find out a theoretical persistenceextinction threshold.

Recently, a lot of population models in a polluted environment have been proposed and investigated; here, we may mention, among many others, [1-23]. Particularly, Yang et al. [15] pointed out that in many cases toxicants should be emitted in regular pulses, for example, the use of pesticides and the pollution by heavy metals (see, e.g., [24]). Thus, they proposed the following two-species LotkaVolterra predator-prey system in a polluted environment with impulsive toxicant input:

$$
\begin{aligned}
& \frac{d x_{1}(t)}{d t}=x_{1}(t)\left[r_{10}-r_{11} C_{10}(t)-a_{11} x_{1}(t)-a_{12} x_{2}(t)\right] \\
& \frac{d x_{2}(t)}{d t}=x_{2}(t)\left[-r_{20}-r_{21} C_{20}(t)+a_{21} x_{1}(t)-a_{22} x_{2}(t)\right], \\
& \frac{d C_{10}(t)}{d t}=k_{1} C_{e}(t)-\left(g_{1}+m_{1}\right) C_{10}(t)
\end{aligned}
$$

$$
\begin{aligned}
& \frac{d C_{20}(t)}{d t}=k_{2} C_{e}(t)-\left(g_{2}+m_{2}\right) C_{20}(t), \\
& \frac{d C_{e}(t)}{d t}=-h C_{e}(t), \\
& t \neq n \gamma, \quad n \in Z^{+}, \\
& \Delta x_{i}(t)=0, \quad \Delta C_{i 0}(t)=0, \quad \Delta C_{e}(t)=b, \\
& t=n \gamma, \quad n \in Z^{+}, \quad i=1,2,
\end{aligned}
$$

where all the parameters are positive constants and $\Delta f(t)=$ $f\left(t^{+}\right)-f(t), Z^{+}=\{1,2, \ldots\} ; x_{1}(t)$ and $x_{2}(t)$ : the size of prey population and the predator population, respectively; $r_{i 0}$ : the intrinsic growth rate of the $i$ th population without toxicant; $r_{i 1}$ : the $i$ th population response to the pollutant present in the organism; $C_{i 0}(t)$ : the concentration of toxicant in the ith organism; $C_{e}(t)$ : the concentration of toxicant in the environment; $k C_{e}(t)$ : the organism's net uptake of toxicant from the environment; $g C_{i 0}(t)+m C_{i 0}(t)$ : the egestion and depuration rates of the toxicant in the $i$ th organism; $h C_{e}(t)$ : the toxicant loss from the environment itself by volatilization and so on; $\gamma$ : the period of the impulsive effect about the exogenous input of toxicant; $b$ : the toxicant input amount at every time.

Yang et al. [15] showed that in the following Lemma holds. 
Lemma 1. For system (1), define

$$
\begin{gathered}
\Delta_{2}=r_{10} a_{21}-r_{20} a_{11}, \quad \bar{\Delta}_{2}:=\frac{a_{21} r_{11} K_{1}}{\gamma}+\frac{a_{11} r_{21} K_{2}}{\gamma}, \\
K_{i}=\frac{k_{i} b}{h\left(g_{i}+m_{i}\right)} .
\end{gathered}
$$

(a) If $r_{10}<r_{11} K_{1} / \gamma$, then $\lim _{t \rightarrow+\infty} x_{i}(t)=0, i=1,2$.

(b) If $r_{10}>r_{11} K_{1} / \gamma$ and $\Delta_{2}<\bar{\Delta}_{2}$, then limsup $\sup _{t \rightarrow+\infty}$ $t^{-1} \int_{0}^{t} x_{1}(s) d s>0$ and $x_{2}(t)$ goes to extinction.

(c) If $\Delta_{2}>\bar{\Delta}_{2}$, then $\lim \sup _{t \rightarrow+\infty} t^{-1} \int_{0}^{t} x_{i}(s) d s>0, i=$ 1,2 .

Some interesting and important problems arise naturally.

(Q1) In the real world, the growth of species depends on various environmental factors, such as temperature, humidity and parasites and so forth. Therefore population models should be stochastic rather than deterministic (May [25]). Thus, what happens if model (1) is subject to stochastic noises?

(Q2) In addition, time delays occur in almost every situation. Kuang [26] has pointed out that ignoring time delays means ignoring reality. Therefore, what happens if model (1) takes time delays into account?

(Q3) Can we improve the results given in Lemma 1?

The aim of this paper is to study the above problems. Suppose that stochastic noises mainly affect the growth rates, with $r_{i 0} \rightarrow r_{i 0}+\alpha_{i} \dot{B}_{i}(t)$ (see, e.g., [27-39]), where $\dot{B}_{i}(t)$ is a white noise and $\alpha_{i}^{2}$ is the intensity of the noise. Moreover, taking time delays into account, we obtain the following model:

$$
\begin{aligned}
& d x_{1}(t)=x_{1}(t)\left[r_{10}-r_{11} C_{10}(t)-a_{11} x_{1}(t)\right. \\
& \left.-a_{12} x_{2}\left(t-\tau_{1}\right)\right] d t+\alpha_{1} x_{1}(t) d B_{1}(t), \\
& d x_{2}(t)=x_{2}(t)\left[-r_{20}-r_{21} C_{20}(t)-a_{22} x_{2}(t)\right. \\
& \left.+a_{21} x_{1}\left(t-\tau_{2}\right)\right] d t+\alpha_{2} x_{2}(t) d B_{2}(t), \\
& \frac{d C_{10}(t)}{d t}=k_{1} C_{e}(t)-\left(g_{1}+m_{1}\right) C_{10}(t), \\
& \frac{d C_{20}(t)}{d t}=k_{2} C_{e}(t)-\left(g_{2}+m_{2}\right) C_{20}(t), \\
& \frac{d C_{e}(t)}{d t}=-h C_{e}(t), \\
& t \neq n \gamma, \quad n \in Z^{+}, \\
& \Delta x_{i}(t)=0, \quad \Delta C_{i 0}(t)=0, \\
& \Delta C_{e}(t)=b, \quad t=n \gamma, n \in Z^{+}, i=1,2,
\end{aligned}
$$

with initial condition

$$
x_{i}(t)=\phi_{i}(t)>0, \quad t \in[-\tau, 0] ; \phi_{i}(0)>0, i=1,2,
$$

where $\tau_{i} \geq 0, \tau=\max \left\{\tau_{1}, \tau_{2}\right\}, \phi_{i}(t)$ is continuous on $[-\tau, 0]$. Our main result is the following theorem.

Theorem 2. For system (3), define

$$
\begin{gathered}
\theta_{1}=r_{10}-0.5 \alpha_{1}^{2}, \quad \theta_{2}=r_{20}+0.5 \alpha_{2}^{2}, \quad \Delta=a_{11} a_{22}+a_{12} a_{21}, \\
\widetilde{\Delta}_{1}:=\theta_{1} a_{22}+\theta_{2} a_{12}, \quad \widetilde{\Delta}_{2}:=\theta_{1} a_{21}-\theta_{2} a_{11}, \\
\bar{\Delta}_{1}:=\frac{a_{22} r_{11} K_{1}}{\gamma}-\frac{a_{12} r_{21} K_{2}}{\gamma} .
\end{gathered}
$$

(i) If $\theta_{1}<r_{11} K_{1} / \gamma$, then both $x_{1}$ and $x_{2}$ go to extinction almost surely (a.s.); that is, $\lim _{t \rightarrow+\infty} x_{i}(t)=0$ a.s., $i=$ $1,2$.

(ii) If $\theta_{1}>r_{11} K_{1} / \gamma$ and $\tilde{\Delta}_{2}<\bar{\Delta}_{2}$, then $x_{2}(t)$ goes to extinction and $x_{1}$ is stable in time average a.s.; that is,

$$
\lim _{t \rightarrow+\infty} t^{-1} \int_{0}^{t} x_{1}(s) d s=\frac{\theta_{1}-r_{11} K_{1} / \gamma}{a_{11}}>0 \text {, a.s. }
$$

(iii) If $\widetilde{\Delta}_{2}>\bar{\Delta}_{2}$, then both $x_{1}$ and $x_{2}$ are stable in time average a.s.

$$
\begin{aligned}
& \lim _{t \rightarrow+\infty} t^{-1} \int_{0}^{t} x_{1}(s) d s=\frac{\widetilde{\Delta}_{1}-\bar{\Delta}_{1}}{\Delta}>0, \text { a.s. } \\
& \lim _{t \rightarrow+\infty} t^{-1} \int_{0}^{t} x_{2}(s) d s=\frac{\widetilde{\Delta}_{2}-\bar{\Delta}_{2}}{\Delta}>0, \text { a.s. }
\end{aligned}
$$

Remark 3. By comparing Lemma 1 with our Theorem 2, we can see that on the one hand, if $\alpha_{1}=\alpha_{2}=0$ and $\tau_{1}=\tau_{2}=0$, then $\theta_{i}=r_{i 0}, \widetilde{\Delta}_{i}=\Delta_{i}, i=1,2$, and our stochastic delay system (3) becomes model (1); on the other hand, our results in Theorem 2 improve that in Lemma 1. Lemma 1 shows that the superior limit is positive, while Theorem 2 reveals that the limit exists and gives the explicit form of the limit. The contribution of this paper is therefore clear.

\section{Proof}

For the sake of simplicity, we introduce some notations:

$$
\begin{gathered}
R_{+}^{2}=\left\{a=\left(a_{1}, a_{2}\right) \in R^{2} \mid a_{i}>0, i=1,2\right\}, \\
\langle f(t)\rangle=t^{-1} \int_{0}^{t} f(s) d s ; \\
\langle f(t)\rangle^{*}=\limsup _{t \rightarrow+\infty} t^{-1} \int_{0}^{t} f(s) d s, \\
\langle f(t)\rangle_{*}=\liminf _{t \rightarrow+\infty} t^{-1} \int_{0}^{t} f(s) d s .
\end{gathered}
$$

Lemma 4. For any given initial value $\phi(t)=\left(\phi_{1}(t), \phi_{2}(t)\right) \epsilon$ $C\left([-\tau, 0], R_{+}^{2}\right)$, there is a unique global positive solution $x(t)=$ $\left(x_{1}(t), x_{2}(t)\right)^{T}$ to the first two equations of system (3) a.s. 
Proof. The proof is similar to Hung [29] by defining

$$
\begin{aligned}
V(x)= & {\left[x_{1}-1-\ln x_{1}\right]+\frac{a_{11} a_{22}}{a_{21}^{2}}\left[x_{2}-1-\ln x_{2}\right] } \\
& +0.5 a_{11} \int_{t-\tau_{2}}^{t} x_{1}^{2}(s) d s
\end{aligned}
$$

and hence is omitted. (3):

To begin with, let us consider the following subsystem of

$$
\begin{gathered}
\frac{d C_{10}(t)}{d t}=k_{1} C_{e}(t)-\left(g_{1}+m_{1}\right) C_{10}(t), \\
\frac{d C_{20}(t)}{d t}=k_{2} C_{e}(t)-\left(g_{2}+m_{2}\right) C_{20}(t), \\
\frac{d C_{e}(t)}{d t}=-h C_{e}(t), \\
t \neq n \gamma, \quad n \in Z^{+}, \\
\Delta C_{10}(t)=0, \quad \Delta C_{20}(t)=0, \quad \Delta C_{e}(t)=b, \\
t=n \gamma, \quad n \in Z^{+}, \\
0 \leq C_{0}(0) \leq 1, \quad 0 \leq C_{e}(0) \leq 1 .
\end{gathered}
$$

Lemma 5 (see $[13,15])$. System (10) has a unique positive $\gamma$ periodic solution $\left(\widetilde{C}_{10}(t), \widetilde{C}_{20}(t), \widetilde{C}_{e}(t)\right)^{T}$, and for each solution $\left(C_{10}(t), C_{20}(t), C_{e}(t)\right)^{T}$ of $(10), C_{10}(t) \rightarrow \widetilde{C}_{10}(t), C_{20}(t) \rightarrow$ $\widetilde{C}_{20}(t)$, and $C_{e}(t) \rightarrow \widetilde{C}_{e}(t)$ as $t \rightarrow \infty$. Moreover, $C_{i 0}(t)>$ $\widetilde{C}_{i 0}(t)$ and $C_{e}(t)>\widetilde{C}_{e}(t)$ for all $t \geq 0$ if $C_{i 0}(0)>\widetilde{C}_{i 0}(0)$ and $C_{e}(0)>\widetilde{C}_{e}(0), i=1,2$, where

$$
\begin{gathered}
\widetilde{C}_{10}(t)=\widetilde{C}_{10}(0) e^{-\left(g_{1}+m_{1}\right)(t-n \gamma)} \\
+\frac{k_{1} b\left(e^{-\left(g_{1}+m_{1}\right)(t-n \gamma)}-e^{-h(t-n \gamma)}\right)}{\left(h-g_{1}-m_{1}\right)\left(1-e^{-h \gamma}\right)}, \\
\widetilde{C}_{20}(t)=\widetilde{C}_{20}(0) e^{-\left(g_{2}+m_{2}\right)(t-n \gamma)} \\
+\frac{k_{2} b\left(e^{-\left(g_{2}+m_{2}\right)(t-n \gamma)}-e^{-h(t-n \gamma)}\right)}{\left(h-g_{2}-m_{2}\right)\left(1-e^{-h \gamma}\right)}, \\
\widetilde{C}_{10}(0)=\frac{\widetilde{C}_{e}(t)=\frac{b e^{-h(t-n \gamma)}}{1-e^{-h \gamma}},}{\left(h-g_{1}-m_{1}\right)\left(1-e^{-\left(g_{1}+m_{1}\right) \gamma}\right)\left(1-e^{-h \gamma}\right)}, \\
\widetilde{C}_{20}(0)=\frac{k_{2} b\left(e^{-\left(g_{1}+m_{1}\right) \gamma}-e^{-h \gamma}\right)}{\left(h-g_{2}-m_{2}\right)\left(1-e^{-\left(g_{2}+m_{2}\right) \gamma}\right)\left(1-e^{-h \gamma}\right)}, \\
\widetilde{C}_{e}(0)=\frac{b}{1-e^{-h \gamma}}
\end{gathered}
$$

for $t \in(n \gamma,(n+1) \gamma]$ and $n \in Z^{+}$. In addition,

$$
\lim _{t \rightarrow+\infty} t^{-1} \int_{0}^{t} \widetilde{C}_{i 0}(s) d s=\frac{k_{i} b}{h\left(g_{i}+m_{i}\right) \gamma}=\frac{K_{i}}{\gamma}, \quad i=1,2 .
$$

Lemma 6 (see [34]). Suppose that $x(t) \in C\left[\Omega \times[0,+\infty), R_{+}\right]$.

(I) If there exist $\sigma$ and positive constants $\sigma_{0}$, T such that

$$
\ln x(t) \leq \sigma t-\sigma_{0} \int_{0}^{t} x(s) d s+\sum_{i=1}^{n} \beta_{i} B_{i}(t)
$$

for $t \geq T$, where $B_{i}(t)$ are independent standard Brownian motions and $\beta_{i}$ are constants, $1 \leq i \leq n$, then one has the following: if $\sigma \geq 0$, then $\langle x\rangle^{*} \leq \sigma / \sigma_{0}$ a.s.; if $\sigma<0$, then $\lim _{t \rightarrow+\infty} x(t)=0$ a.s.

(II) If there exist positive constants $\sigma_{0}, T$ and $\sigma$ such that

$$
\ln x(t) \geq \sigma t-\sigma_{0} \int_{0}^{t} x(s) d s+\sum_{i=1}^{n} \beta_{i} B_{i}(t)
$$

for $t \geq T$, then $\langle x\rangle_{*} \geq \sigma / \sigma_{0}$ a.s.

Now, let us consider the following auxiliary system:

$$
\begin{array}{r}
d y_{1}(t)=y_{1}(t)\left[r_{10}-r_{11} C_{10}(t)-a_{11} y_{1}(t)\right] d t \\
+\alpha_{1} y_{1}(t) d B_{1}(t), \\
d y_{2}(t)=y_{2}(t)\left[-r_{20}-r_{21} C_{20}(t)+a_{21} y_{1}\left(t-\tau_{2}\right)\right. \\
\left.\quad-a_{22} y_{2}(t)\right] d t+\alpha_{2} y_{2}(t) d B_{2}(t),
\end{array}
$$

with initial value $\phi(t) \in C\left([-\tau, 0], R_{+}^{2}\right)$.

Lemma 7. If $\theta_{1}=r_{10}-0.5 \alpha_{1}^{2}>r_{11} K_{1} / \gamma$, then the solution $y(t)$ of system (15) obeys

$$
\begin{gathered}
\lim _{t \rightarrow+\infty}\left\langle y_{1}(t)\right\rangle=\frac{\theta_{1}-r_{11} K_{1} / \gamma}{a_{11}} ; \\
\lim _{t \rightarrow+\infty} y_{2}(t)=0 \text { a.s., if } \widetilde{\Delta}_{2}<\bar{\Delta}_{2} ; \\
\lim _{t \rightarrow+\infty}\left\langle y_{2}(t)\right\rangle=\frac{\widetilde{\Delta}_{2}-\bar{\Delta}_{2}}{a_{11} a_{22}} \text { a.s., if } \widetilde{\Delta}_{2}>\bar{\Delta}_{2} .
\end{gathered}
$$

Proof. By Lemma 5,

$$
\begin{aligned}
\lim _{t \rightarrow+\infty} t^{-1} \int_{0}^{t} C_{i 0}(s) d s & =\lim _{t \rightarrow+\infty} t^{-1} \int_{0}^{t} \widetilde{C}_{i 0}(s) d s \\
& =\frac{K_{i}}{\gamma}, \quad i=1,2 .
\end{aligned}
$$

Then, for all $\varepsilon>0$, there exists $T>0$ such that

$$
\frac{K_{i}}{\gamma}-\varepsilon \leq\left\langle C_{i 0}(t)\right\rangle \leq \frac{K_{i}}{\gamma}+\varepsilon, \quad t>T, i=1,2 .
$$


An application of Itô's formula to (15) yields

$$
\begin{aligned}
& \ln y_{1}(t)-\ln y_{1}(0) \\
&= \theta_{1} t-r_{11} \int_{0}^{t} C_{10}(s) d s-a_{11} \int_{0}^{t} y_{1}(s) d s+\alpha_{1} B_{1}(t) \\
& \ln y_{2}(t)-\ln y_{2}(0) \\
&=-\theta_{2} t-r_{21} \int_{0}^{t} C_{20}(s) d s \\
& \quad+a_{21} \int_{0}^{t} y_{1}\left(s-\tau_{2}\right) d s-a_{22} \int_{0}^{t} y_{2}(s) d s+\alpha_{2} B_{2}(t) \\
&=-\theta_{2} t-r_{21} \int_{0}^{t} C_{20}(s) d s+a_{21} \int_{0}^{t} y_{1}(s) d s \\
& \quad-a_{21}\left[\int_{t-\tau_{2}}^{t} y_{1}(s) d s-\int_{-\tau_{2}}^{0} y_{1}(s) d s\right] \\
& \quad-a_{22} \int_{0}^{t} y_{2}(s) d s+\alpha_{2} B_{2}(t)
\end{aligned}
$$

That is to say, we have shown that

$$
\begin{gathered}
t^{-1} \ln \frac{y_{1}(t)}{y_{1}(0)}=\theta_{1}-r_{11}\left\langle C_{10}(t)\right\rangle-a_{11}\left\langle y_{1}(t)\right\rangle+t^{-1} \alpha_{1} B_{1}(t) \\
t^{-1} \ln \frac{y_{2}(t)}{y_{2}(0)}+t^{-1} a_{21}\left[\int_{t-\tau_{2}}^{t} y_{1}(s) d s-\int_{-\tau_{2}}^{0} y_{1}(s) d s\right] \\
=-\theta_{2}-r_{21}\left\langle C_{20}(t)\right\rangle+a_{21}\left\langle y_{1}(t)\right\rangle \\
-a_{22}\left\langle y_{2}(t)\right\rangle+t^{-1} \alpha_{2} B_{2}(t) .
\end{gathered}
$$

When (18) is used in (20), we can see that for $t>T$,

$$
\begin{aligned}
t^{-1} \ln \frac{y_{1}(t)}{y_{1}(0)} \leq & \theta_{1}-\frac{r_{11} K_{1}}{\gamma}+r_{11} \varepsilon \\
& -a_{11}\left\langle y_{1}(t)\right\rangle+t^{-1} \alpha_{1} B_{1}(t), \\
t^{-1} \ln \frac{y_{1}(t)}{y_{1}(0)} \geq & \theta_{1}-\frac{r_{11} K_{1}}{\gamma}-r_{11} \varepsilon \\
& -a_{11}\left\langle y_{1}(t)\right\rangle+t^{-1} \alpha_{1} B_{1}(t) .
\end{aligned}
$$

Let $\varepsilon$ be sufficiently small such that $\theta_{1}-r_{11} K_{1} / \gamma-r_{11} \varepsilon>0$. Making use of (I) and (II) in Lemma 6 to (22) and (23), respectively, we have

$$
\begin{aligned}
& \left\langle y_{1}(t)\right\rangle^{*} \leq \frac{\theta_{1}-r_{11} K_{1} / \gamma+r_{11} \varepsilon}{a_{11}}, \\
& \left\langle y_{1}(t)\right\rangle_{*} \geq \frac{\theta_{1}-r_{11} K_{1} / \gamma-r_{11} \varepsilon}{a_{11}} .
\end{aligned}
$$

It then follows from the arbitrariness of $\varepsilon$ that

$$
\lim _{t \rightarrow+\infty}\left\langle y_{1}(t)\right\rangle=\frac{\theta_{1}-r_{11} K_{1} / \gamma}{a_{11}} .
$$

Substituting (17) and (25) into (20) and noting that $\lim _{t \rightarrow+\infty}$ $t^{-1} B_{1}(t)=0$, one can derive that

$$
\lim _{t \rightarrow+\infty} t^{-1} \ln y_{1}(t)=0, \text { a.s. }
$$

Employing (20) and (21) in the expression $a_{21} \ln \left(y_{1}(t) /\right.$ $\left.y_{1}(0)\right)+a_{11} \ln \left(y_{2}(t) / y_{2}(0)\right)$ yields

$$
\begin{aligned}
a_{11} t^{-1} \ln & \frac{y_{2}(t)}{y_{2}(0)}+a_{21} t^{-1} \ln \frac{y_{1}(t)}{y_{1}(0)} \\
= & \widetilde{\Delta}_{2}-r_{11} a_{21}\left\langle C_{10}(t)\right\rangle-r_{21} a_{11}\left\langle C_{20}(t)\right\rangle \\
& -a_{11} a_{22}\left\langle y_{2}(t)\right\rangle \\
& -t^{-1} a_{11} a_{21}\left[\int_{t-\tau_{2}}^{t} y_{1}(s) d s-\int_{-\tau_{2}}^{0} y_{1}(s) d s\right] \\
& +t^{-1}\left[a_{21} \alpha_{1} B_{1}(t)+a_{11} \alpha_{2} B_{2}(t)\right] .
\end{aligned}
$$

In view of (25), we get

$$
\begin{aligned}
\lim _{t \rightarrow+\infty} t^{-1} \int_{t-\tau_{2}}^{t} y_{1}(s) d s \\
\quad=\lim _{t \rightarrow+\infty} t^{-1}\left(\int_{0}^{t} y_{1}(s) d s-\int_{0}^{t-\tau_{2}} y_{1}(s) d s\right)=0 \text {, a.s. }
\end{aligned}
$$

By (17), (26), (27), and (28), for all $\varepsilon>0$, there exists $T>0$ such that, for $t \geq T$,

$$
\begin{aligned}
a_{11} t^{-1} \ln \frac{y_{2}(t)}{y_{2}(0)} \leq & \widetilde{\Delta}_{2}-\bar{\Delta}_{2}+\varepsilon-a_{11} a_{22}\left\langle y_{2}(t)\right\rangle \\
& +t^{-1}\left[a_{21} \sigma_{1} B_{1}(t)+a_{11} \sigma_{2} B_{2}(t)\right], \\
a_{11} t^{-1} \ln \frac{y_{2}(t)}{y_{2}(0)} \geq & \widetilde{\Delta}_{2}-\bar{\Delta}_{2}-\varepsilon-a_{11} a_{22}\left\langle y_{2}(t)\right\rangle \\
& +t^{-1}\left[a_{21} \sigma_{1} B_{1}(t)+a_{11} \sigma_{2} B_{2}(t)\right] .
\end{aligned}
$$

If $\widetilde{\Delta}_{2}<\bar{\Delta}_{2}$, then we can choose $\varepsilon$ sufficiently small such that $\widetilde{\Delta}_{2}-\bar{\Delta}_{2}+\varepsilon<0$. Then, by (29) and (I) in Lemma 6, we obtain $\lim _{t \rightarrow+\infty} y_{2}(t)=0$ a.s. If $\widetilde{\Delta}_{2}>\bar{\Delta}_{2}$, then we can choose $\varepsilon$ sufficiently small such that $\widetilde{\Delta}_{2}-\bar{\Delta}_{2}-\varepsilon>0$. An application of (I) and (II) in Lemma 6 to (29) and (30), respectively, makes one observe that

$$
\frac{\widetilde{\Delta}_{2}-\bar{\Delta}_{2}-\varepsilon}{a_{11} a_{22}} \leq\left\langle y_{2}(t)\right\rangle_{*} \leq\left\langle y_{2}(t)\right\rangle^{*} \leq \frac{\widetilde{\Delta}_{2}-\bar{\Delta}_{2}+\varepsilon}{a_{11} a_{22}} \text {, a.s. }
$$

Therefore, using the arbitrariness of $\varepsilon$ results in

$$
\lim _{t \rightarrow+\infty}\left\langle y_{2}(t)\right\rangle=\frac{\widetilde{\Delta}_{2}-\bar{\Delta}_{2}}{a_{11} a_{22}} \text { a.s. }
$$

This completes the proof.

We are now in the position to prove our main results. 
Proof of Theorem 2. Applying Itô's formula to (3) leads to

$$
\begin{aligned}
& \ln x_{1}(t)-\ln x_{1}(0) \\
&= \theta_{1} t-r_{11} \int_{0}^{t} C_{10}(s) d s-a_{11} \int_{0}^{t} x_{1}(s) d s \\
&-a_{12} \int_{0}^{t} x_{2}\left(s-\tau_{1}\right) d s+\alpha_{1} B_{1}(t) \\
&= \theta_{1} t-r_{11} \int_{0}^{t} C_{10}(s) d s-a_{12} \int_{0}^{t} x_{2}(s) d s \\
&+a_{12}\left[\int_{t-\tau_{1}}^{t} x_{2}(s) d s-\int_{-\tau_{1}}^{0} x_{2}(s) d s\right] \\
&-a_{11} \int_{0}^{t} x_{1}(s) d s+\alpha_{1} B_{1}(t) . \\
& \ln x_{2}(t)-\ln x_{2}(0) \\
&=-\theta_{2} t-r_{21} \int_{0}^{t} C_{20}(s) d s+a_{21} \int_{0}^{t} x_{1}(s) d s \\
&-a_{21}\left[\int_{t-\tau_{2}}^{t} x_{1}(s) d s-\int_{-\tau_{2}}^{t} x_{1}(s) d s\right] \\
& x_{2}(s) d s+\alpha_{2} B_{2}(t) .
\end{aligned}
$$

(i) It follows from (17) and (33) that

$$
\begin{aligned}
& t^{-1} \ln x_{1}(t)-t^{-1} \ln x_{1}(0) \\
& \quad \leq \theta_{1}-r_{11}\left\langle C_{10}(t)\right\rangle-a_{11}\left\langle x_{1}(t)\right\rangle+\frac{\alpha_{1} B_{1}(t)}{t} \\
& \quad \leq \theta_{1}-\frac{r_{11} K_{1}}{\lambda}+\varepsilon-a_{11}\left\langle x_{1}(t)\right\rangle+\frac{\alpha_{1} B_{1}(t)}{t}
\end{aligned}
$$

for sufficiently large $t$. Since $\theta_{1}-r_{11} K_{1} / \lambda<0$, then we can choose $\varepsilon$ sufficiently small such that $\theta_{1}-r_{11} K_{1} / \lambda+\varepsilon<0$. Then, by (I) in Lemma 6,

$$
\lim _{t \rightarrow+\infty} x_{1}(t)=0 \text {, a.s. }
$$

When (36) is used in (34), one can see that

$$
t^{-1} \ln x_{2}(t)-\ln x_{2}(0) \leq-\theta_{2}+\varepsilon-a_{22}\left\langle x_{2}(t)\right\rangle+\frac{\alpha_{2} B_{2}(t)}{t}
$$

for sufficiently large $t$, where $\varepsilon>0$ obeys $-\theta_{2}+\varepsilon<0$. In view of Lemma 6 again, $\lim _{t \rightarrow+\infty} x_{2}(t)=0$, a.s.

(ii) By the stochastic comparison theorem [40], one can observe that

$$
x_{1}(t) \leq y_{1}(t), \quad x_{2}(t) \leq y_{2}(t)
$$

Note that $\theta_{1}>r_{11} K_{1} / \gamma$ and $\widetilde{\Delta}_{2}<\bar{\Delta}_{2}$; it then follows from Lemma 7 that $\lim _{t \rightarrow+\infty} y_{2}(t)=0$, a.s. Making use of (38) gives $\lim _{t \rightarrow+\infty} x_{2}(t)=0$, a.s. Thus, for all $\varepsilon>0$, there exists $T>0$ such that, for $t \geq T$,

$$
\frac{\varepsilon}{2} \leq a_{12} x_{2}(t) \leq \frac{\varepsilon}{2} .
$$

Substituting the above inequalities into (33) and then using (18), we obtain

$$
\begin{aligned}
t^{-1} \ln x_{1}(t) \leq & t^{-1} \ln x_{1}(0)+\theta_{1}-r_{11}\left\langle C_{10}(t)\right\rangle \\
& -a_{11}\left\langle x_{1}(t)\right\rangle+\frac{\varepsilon}{2}+\frac{\alpha_{1} B_{1}(t)}{t} \\
\leq & \theta_{1}-\frac{r_{11} K_{1}}{\gamma}+2 \varepsilon-a_{11}\left\langle x_{1}(t)\right\rangle+\frac{\alpha_{1} B_{1}(t)}{t}, \\
t^{-1} \ln x_{1}(t) \geq & t^{-1} \ln x_{1}(0)+\theta_{1}-r_{11}\left\langle C_{10}(t)\right\rangle \\
& -a_{11}\left\langle x_{1}(t)\right\rangle-\frac{\varepsilon}{2}+\frac{\alpha_{1} B_{1}(t)}{t} \\
\geq & \theta_{1}-\frac{r_{11} K_{1}}{\gamma}-2 \varepsilon-a_{11}\left\langle x_{1}(t)\right\rangle+\frac{\alpha_{1} B_{1}(t)}{t} .
\end{aligned}
$$

Let $\varepsilon$ be sufficiently small such that $\theta_{1}-r_{11} K_{1} / \gamma-\varepsilon>0$, and then, applying (I) and (II) in Lemma 6 to (40) and (41), respectively, one can see that

$$
\begin{aligned}
\frac{\theta_{1}-r_{11} K_{1} / \gamma-2 \varepsilon}{a_{11}} & \leq\left\langle x_{1}(t)\right\rangle_{*} \leq\left\langle x_{1}(t)\right\rangle^{*} \\
& \leq \frac{\theta_{1}-r_{11} K_{1} / \gamma+2 \varepsilon}{a_{11}} \text { a.s. }
\end{aligned}
$$

An application of the arbitrariness of $\varepsilon$ gives

$$
\lim _{t \rightarrow+\infty}\left\langle x_{1}(t)\right\rangle=\frac{\theta_{1}-r_{11} K_{1} / \gamma}{a_{11}}, \text { a.s. }
$$

(iii) Clearly, $\widetilde{\Delta}_{2}>\bar{\Delta}_{2}$ implies $\theta_{1}>r_{11} K_{1} / \gamma$, and then, by Lemma 7,

$$
\lim _{t \rightarrow+\infty}\left\langle y_{2}(t)\right\rangle=\frac{\tilde{\Delta}_{2}-\bar{\Delta}_{2}}{a_{11} a_{22}} .
$$

Thus, similar to the proof of (28), we get

$$
\lim _{t \rightarrow+\infty} t^{-1} \int_{t-\tau_{1}}^{t} y_{2}(s) d s=0 \text { a.s. }
$$

Therefore, by (26), (28), and (38), we can observe that

$$
\begin{gathered}
\limsup _{t \rightarrow+\infty} t^{-1} \ln x_{1}(t) \leq \lim _{t \rightarrow+\infty} t^{-1} \ln y_{1}(t)=0, \\
\lim _{t \rightarrow+\infty} t^{-1} \int_{t-\tau_{2}}^{t} x_{1}(s) d s=0, \\
\lim _{t \rightarrow+\infty} t^{-1} \int_{t-\tau_{1}}^{t} x_{2}(s) d s=0 \text {, a.s. }
\end{gathered}
$$


Employing (33) and (34) in the expression $a_{21} \ln \left(x_{1}(t) /\right.$ $\left.x_{1}(0)\right)+a_{11} \ln \left(x_{2}(t) / x_{2}(0)\right)$ yields

$$
\begin{aligned}
t^{-1} a_{21} \ln \frac{x_{1}(t)}{x_{1}(0)}+t^{-1} a_{11} \ln \frac{x_{2}(t)}{x_{2}(0)} \\
=a_{12} a_{21} t^{-1}\left[\int_{t-\tau_{1}}^{t} x_{2}(s) d s-\int_{-\tau_{1}}^{0} x_{2}(s) d s\right] \\
\quad-a_{11} a_{21} t^{-1}\left[\int_{t-\tau_{2}}^{t} x_{1}(s) d s-\int_{-\tau_{2}}^{0} x_{1}(s) d s\right] \\
+\widetilde{\Delta}_{2}-a_{21} r_{11}\left\langle C_{10}(t)\right\rangle-a_{11} r_{21}\left\langle C_{20}(t)\right\rangle \\
\quad-\Delta\left\langle x_{2}(t)\right\rangle+t^{-1} a_{21} \alpha_{1} B_{1}(t)+t^{-1} a_{11} \alpha_{2} B_{2}(t) .
\end{aligned}
$$

When (18), (46) and (47), are used in (48), one can obtain

$$
\begin{aligned}
t^{-1} a_{11} \ln \frac{x_{2}(t)}{x_{2}(0)} \geq & \widetilde{\Delta}_{2}-\bar{\Delta}_{2}-\varepsilon-\Delta\left\langle x_{2}(t)\right\rangle \\
& +t^{-1} a_{21} \alpha_{1} B_{1}(t)+t^{-1} a_{11} \alpha_{2} B_{2}(t)
\end{aligned}
$$

for sufficiently large $t$, where $\varepsilon>0$ obeys $\widetilde{\Delta}_{2}-\bar{\Delta}_{2}-\varepsilon>0$. It then follows from (II) in Lemma 6 that

$$
\left\langle x_{2}(t)\right\rangle_{*} \geq \frac{\widetilde{\Delta}_{2}-\bar{\Delta}_{2}-\varepsilon}{\Delta} .
$$

By virtue of the arbitrariness of $\varepsilon$, we can see that

$$
\left\langle x_{2}(t)\right\rangle_{*} \geq \frac{\tilde{\Delta}_{2}-\bar{\Delta}_{2}}{\Delta} .
$$

Consequently, for every $0<\varepsilon<a_{12}\left(\widetilde{\Delta}_{2}-\bar{\Delta}_{2}\right) / \Delta$, there is $T>0$ such that

$$
a_{12}\left\langle x_{2}(t)\right\rangle \geq a_{12}\left\langle x_{2}\right\rangle_{*}-\varepsilon \geq \frac{a_{12}\left(\widetilde{\Delta}_{2}-\bar{\Delta}_{2}\right)}{\Delta}-\varepsilon, \quad t>T .
$$

Substituting the above inequality into (33) and then using (18) and (47), one can see that

$$
\begin{aligned}
t^{-1} \ln \frac{x_{1}(t)}{x_{1}(0)} \leq & \theta_{1}-\frac{a_{12}\left(\widetilde{\Delta}_{2}-\bar{\Delta}_{2}\right)}{\Delta}+3 \varepsilon \\
& -a_{11} t^{-1} \int_{0}^{t} x_{1}(s) d s+t^{-1} \alpha_{1} B_{1}(t) \\
= & \frac{a_{11}\left(\widetilde{\Delta}_{1}-\bar{\Delta}_{1}\right)}{\Delta}+3 \varepsilon-a_{11} t^{-1} \int_{0}^{t} x_{1}(s) d s \\
& +t^{-1} \alpha_{1} B_{1}(t)
\end{aligned}
$$

for sufficiently large $t$. Since $\widetilde{\Delta}_{1}-\bar{\Delta}_{1}>0$, and then, by Lemma 6 and the arbitrariness of $\varepsilon$, one can observe that

$$
\left\langle x_{1}(t)\right\rangle^{*} \leq \frac{\tilde{\Delta}_{1}-\bar{\Delta}_{1}}{\Delta} .
$$

When this inequality, (18) and (47), are used in (34), we can see that

$$
\begin{aligned}
t^{-1} \ln \frac{x_{2}(t)}{x_{2}(0)} \leq & -\theta_{2}+a_{21} \frac{\widetilde{\Delta}_{1}-\bar{\Delta}_{1}}{\Delta}+3 \varepsilon \\
& -a_{22} t^{-1} \int_{0}^{t} x_{2}(s) d s+t^{-1} \alpha_{2} B_{2}(t) \\
= & \frac{a_{22}\left(\widetilde{\Delta}_{2}-\bar{\Delta}_{2}\right)}{\Delta}+3 \varepsilon-a_{22} t^{-1} \int_{0}^{t} x_{2}(s) d s \\
& +t^{-1} \alpha_{2} B_{2}(t)
\end{aligned}
$$

for sufficiently large $t$. Then, it follows from Lemma 6 and the arbitrariness of $\varepsilon$ that

$$
\left\langle x_{2}(t)\right\rangle^{*} \leq \frac{\widetilde{\Delta}_{2}-\bar{\Delta}_{2}}{\Delta}
$$

Substituting the above inequality and (18) into (33), we get

$$
\begin{aligned}
t^{-1} \ln \frac{x_{1}(t)}{x_{1}(0)} \geq & \theta_{1}-a_{12} \frac{\tilde{\Delta}_{2}-\bar{\Delta}_{2}}{\Delta}-3 \varepsilon \\
& -a_{11} t^{-1} \int_{0}^{t} x_{1}(s) d s+t^{-1} \alpha_{1} B_{1}(t) \\
= & \frac{a_{11}\left(\widetilde{\Delta}_{1}-\bar{\Delta}_{1}\right)}{\Delta}-3 \varepsilon-a_{11} t^{-1} \int_{0}^{t} x_{1}(s) d s \\
& +t^{-1} \alpha_{1} B_{1}(t)
\end{aligned}
$$

for sufficiently large $t$. By (II) in Lemma 6 and the arbitrariness of $\varepsilon$ again, we obtain

$$
\left\langle x_{1}(t)\right\rangle_{*} \geq \frac{\widetilde{\Delta}_{1}-\bar{\Delta}_{1}}{\Delta} .
$$

Then, the required assertion follows from (51), (54), (56), and (58).

\section{Numerical Simulations}

Let us use the famous Milstein method (see, e.g., [41]) to illustrate the analytical results.

To begin with, we choose $r_{10}=0.85, r_{20}=0.05, r_{11}=$ $r_{21}=1, a_{11}=0.4, a_{12}=0.4, a_{21}=0.3, a_{22}=0.3, \tau_{1}=3, \tau_{2}=$ $8, \alpha_{2}^{2}=0.1, k_{i}=g_{i}=m_{i}=0.1, i=1,2, h=0.5, b=0.6$, and $\gamma=12$. Then,

$$
\begin{gathered}
K_{i}=\frac{k_{i} b}{h\left(g_{i}+m_{i}\right)}=0.6, \\
\Delta_{2}=r_{10} a_{21}-r_{20} a_{11}=0.235>\bar{\Delta}_{2} \\
=\frac{a_{21} r_{11} K_{1}}{\gamma}+\frac{a_{11} r_{21} K_{2}}{\gamma}=0.035 .
\end{gathered}
$$




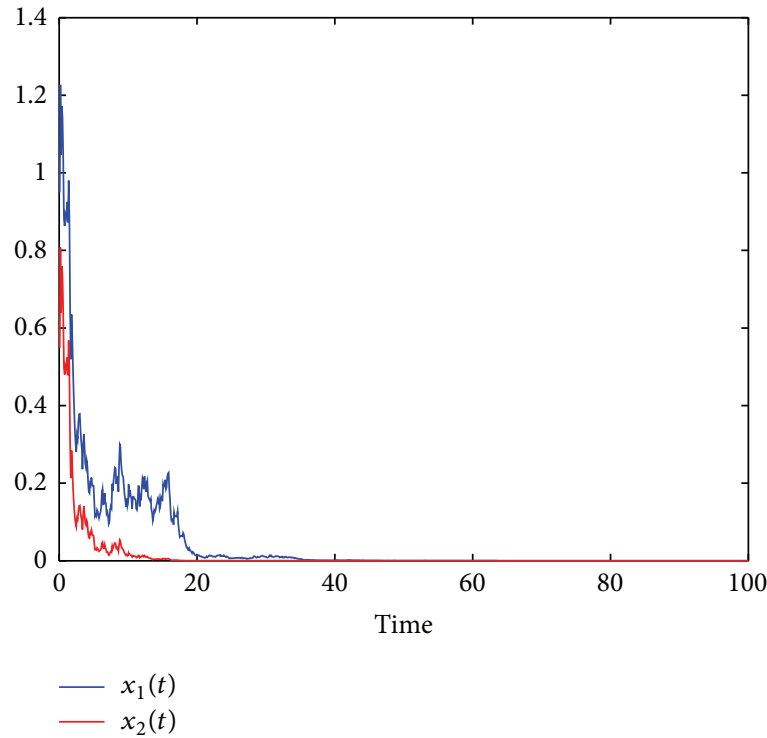

(a)

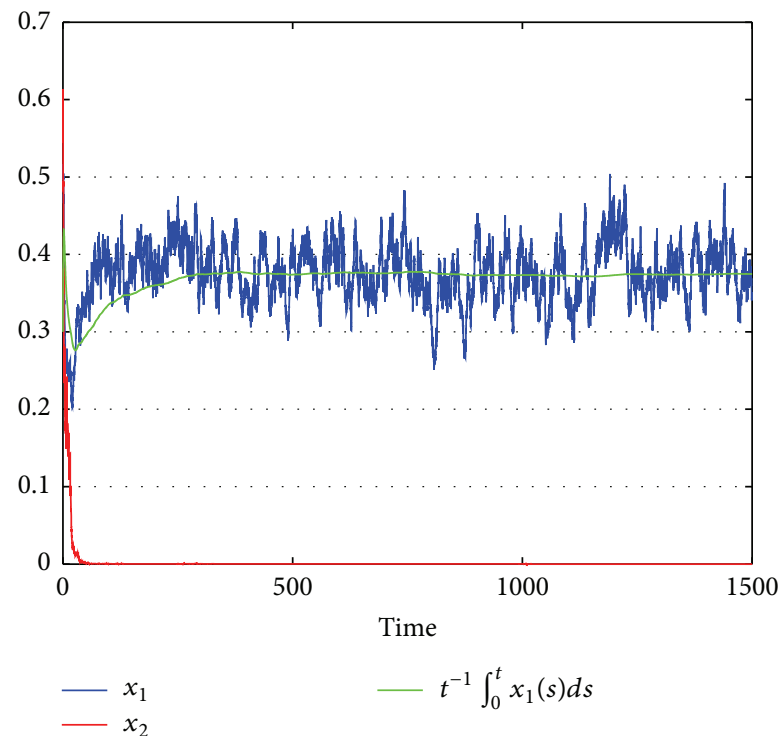

(b)

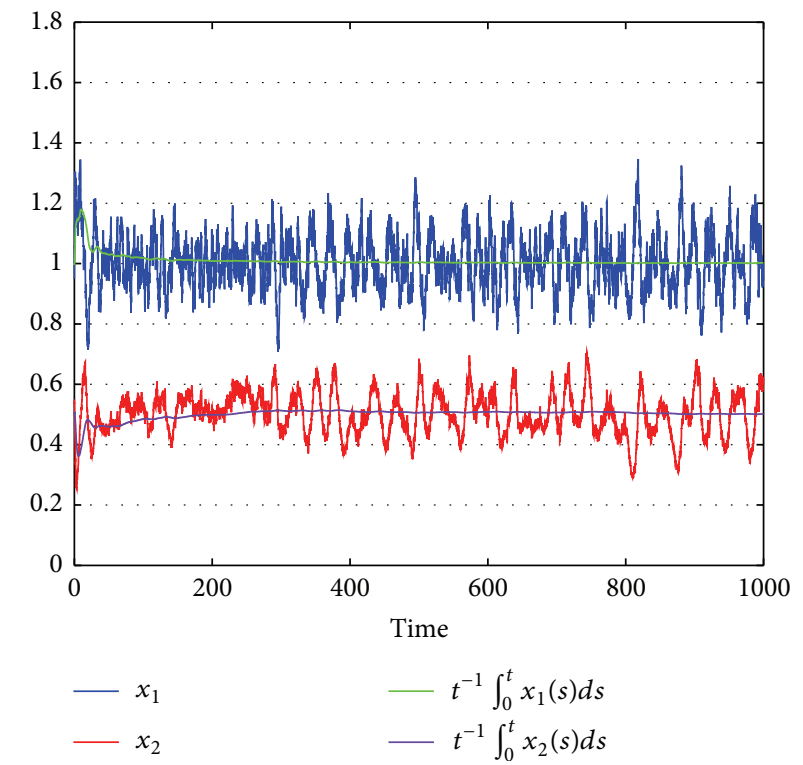

(c)

Figure 1: Solutions of system (3) for $r_{10}=0.85, r_{20}=0.05, r_{11}=r_{21}=1, a_{11}=0.4, a_{12}=0.4, a_{21}=0.3, a_{22}=0.3, \tau_{1}=3, \tau_{2}=8, \alpha_{2}^{2}=0.1$, $k_{i}=g_{i}=m_{i}=0.1, i=1,2, h=0.5, b=0.6, \gamma=12, x_{1}(0)=0.9, x_{2}(0)=0.5, C_{0}(0)=C_{e}(0)=0.1$, and step size $\Delta t=0.001$. (a) is with $\alpha_{1}^{2} / 2=0.82$; (b) is with $\alpha_{1}^{2} / 2=0.65$; (c) is with $\alpha_{1}^{2} / 2=0.2$.

By (c) in Lemma 1, the solution of model (1) obeys

$$
\limsup _{t \rightarrow+\infty} t^{-1} \int_{0}^{t} x_{1}(s) d s>0, \quad \limsup _{t \rightarrow+\infty} t^{-1} \int_{0}^{t} x_{2}(s) d s>0 .
$$

However, when the white noises are taken into account, the properties of the system may be changed greatly. In Figure 1, we let the coefficients be same with the above. The only difference between conditions of Figures 1(a), 1(b), and 1(c) is that the value of $\alpha_{1}^{2}$ is different. In Figure 1(a), we choose $\alpha_{1}^{2} / 2=0.82$. Therefore,

$$
\theta_{1}=r_{10}-\frac{\alpha_{1}^{2}}{2}=0.03<\frac{r_{11} K_{1}}{\gamma}=0.05
$$

Then, by (i) in Theorem 2, both $x_{1}$ and $x_{2}$ are extinctive. Figure 1(a) confirms these. In Figure 1(b), we choose $\alpha_{1}^{2} / 2=$ 0.65. That is to say $\theta_{1}=0.2>r_{11} K_{1} / \gamma=0.05$ and $\widetilde{\Delta}_{2}=0.02<\bar{\Delta}_{2}=0.035$. It then follows from (ii) in 


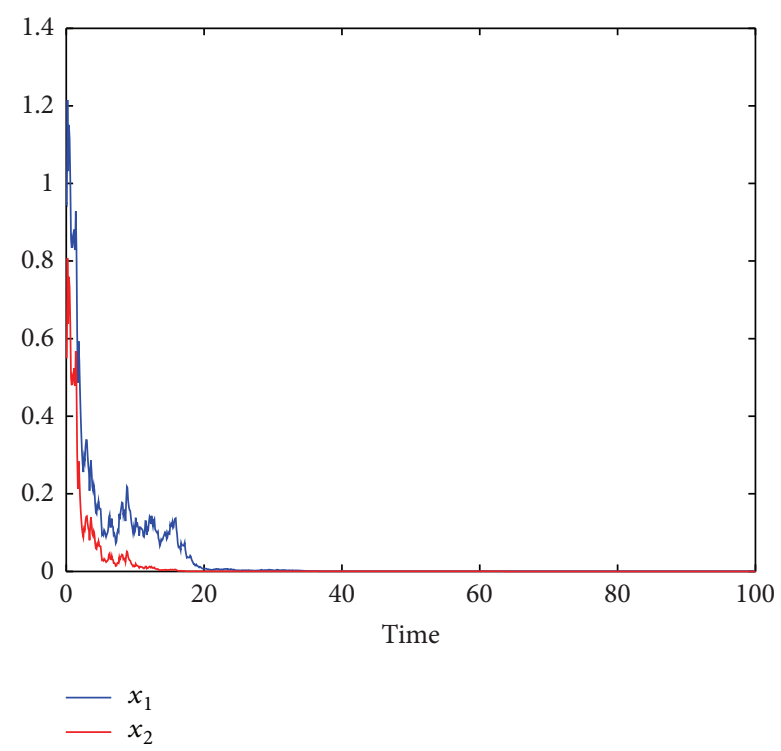

Figure 2: Solutions of system (3) for $r_{10}=0.85, r_{20}=0.05, r_{11}=$ $r_{21}=1, a_{11}=0.4, a_{12}=0.4, a_{21}=0.3, a_{22}=0.3, \tau_{1}=3, \tau_{2}=8$, $\alpha_{1}^{2}=0.4, \alpha_{2}^{2}=0.1, k_{i}=g_{i}=m_{i}=0.1, i=1,2, h=0.5, b=0.6$, $\gamma=0.8, x_{1}(0)=0.9, x_{2}(0)=0.5, C_{0}(0)=C_{e}(0)=0.1$, and step size $\Delta t=0.001$.

Theorem 2 that $x_{2}$ is extinctive and $x_{1}$ is stable in time average:

$$
\lim _{t \rightarrow+\infty}\left\langle x_{1}(t)\right\rangle=\frac{\theta_{1}-r_{11} K_{1} / \gamma}{a_{11}}=0.375
$$

See Figure 1(b). In Figure 1(c), we choose $\alpha_{1}^{2} / 2=0.2$. Then, $\widetilde{\Delta}_{2}=0.155>\bar{\Delta}_{2}=0.035$. In view of (iii) in Theorem 2, we can obtain that both $x_{1}$ and $x_{2}$ are stable in time average:

$$
\begin{aligned}
& \lim _{t \rightarrow+\infty}\left\langle x_{1}(t)\right\rangle=\frac{\widetilde{\Delta}_{1}-\bar{\Delta}_{1}}{\Delta}=\frac{0.24}{0.24}=1, \\
& \lim _{t \rightarrow+\infty}\left\langle x_{2}(t)\right\rangle=\frac{\widetilde{\Delta}_{2}-\bar{\Delta}_{2}}{\Delta}=\frac{0.12}{0.24}=0.5 .
\end{aligned}
$$

Figure 1(c) confirms these.

In Figure 2, we choose $r_{10}=0.85, r_{20}=0.05, r_{11}=$ $r_{21}=1, a_{11}=0.4, a_{12}=0.4, a_{21}=0.3, a_{22}=0.3, \tau_{1}=3$, $\tau_{2}=8, \alpha_{1}^{2}=0.4, \alpha_{2}^{2}=0.1, k_{i}=g_{i}=m_{i}=0.1, i=1,2$, $h=0.5$, and $b=0.6$. The only difference between conditions of Figures $1(\mathrm{c})$ and 2 is that the value of $\gamma$ is different. In Figure 2, we choose $\gamma=0.8$. Then, $\theta_{1}=0.65<r_{11} K_{1} / \gamma=$ 0.75 . It follows from (i) in Theorem 2 that both $x_{1}$ and $x_{2}$ are extinctive. Figure 2 confirms these. By comparing Figure 1(c) with Figure 2, one can see that the impulsive period $\gamma$ plays a key role in determining the stability in time average and the extinction of the species.

\section{Conclusions and Future Directions}

This paper is concerned with stochastic delay predator-prey model in a polluted environment with impulsive toxicant input. For each species, the threshold between stability in time average and extinction is established. Some recent results are improved and extended. Our Theorem 2 reveals some interesting and important results.

(A) Firstly, time delay is harmless for stability in time average and extinction of the stochastic system (3).

(B) The white noise $\alpha_{1} d B_{1}(t)$ and $\alpha_{2} d B_{2}(t)$ can change the properties of the system greatly.

(C) The impulsive period $\gamma$ plays an important role in determining the stability in time average and the extinction of the species.

Some interesting questions deserve further investigations. One may consider some more realistic but more complex systems, for example, stochastic delay model with Markov switching (see, e.g., [30, 32, 39]). It is also interesting to investigate what happens if $a_{i j}$ is stochastic.

\section{Acknowledgments}

The author thanks the editor and reviewer for these valuable and important comments. This research is supported by NSFC of China (nos. 11301207, 11171081, 11301112 and 11171056), Natural Science Foundation of Jiangsu Province (No. BK20130411) and Natural Science Research Project of Ordinary Universities in Jiangsu Province (no. 13KJB110002).

\section{References}

[1] T. G. Hallam, C. E. Clark, and R. R. Lassiter, "Effects of toxicants on populations: a qualitative approach-1. Equilibrium environmental exposure," Ecological Modelling, vol. 18, no. 4, pp. 291-304, 1983.

[2] T. G. Hallam, C. E. Clark, and G. S. Jordan, "Effects of toxicants on populations: a qualitative approach-2. First order kinetics," Journal of Mathematical Biology, vol. 18, no. 1, pp. 25-37, 1983.

[3] T. G. Hallam and J. T. De Luna, "Effects of toxicants on populations: a qualitative approach-3. Environmental and food chain pathways," Journal of Theoretical Biology, vol. 109, no. 3, pp. 411-429, 1984.

[4] Z. E. Ma, G. R. Cui, and W. D. Wang, "Persistence and extinction of a population in a polluted environment," Mathematical Biosciences, vol. 101, no. 1, pp. 75-97, 1990.

[5] H. I. Freedman and J. B. Shukla, "Models for the effect of toxicant in single-species and predator-prey systems," Journal of Mathematical Biology, vol. 30, no. 1, pp. 15-30, 1991.

[6] L. Huaping and M. Zhien, "The threshold of survival for system of two species in a polluted environment," Journal of Mathematical Biology, vol. 30, no. 1, pp. 49-61, 1991.

[7] M. Zhien, Z. Wengang, and L. Zhixue, "The thresholds of survival for an $n$-food chain model in a polluted environment," Journal of Mathematical Analysis and Applications, vol. 210, no. 2, pp. 440-458, 1997.

[8] B. Buonomo, A. Di Liddo, and I. Sgura, "A diffusive-convective model for the dynamics of population-toxicant interactions: some analytical and numerical results," Mathematical Biosciences, vol. 157, no. 1-2, pp. 37-64, 1999. 
[9] P. Jinxiao, J. Zhen, and M. Zhien, "Thresholds of survival for an $n$-dimensional Volterra mutualistic system in a polluted environment," Journal of Mathematical Analysis and Applications, vol. 252, no. 2, pp. 519-531, 2000.

[10] Z. Li, Z. Shuai, and K. Wang, "Persistence and extinction of single population in a polluted environment," Electronic Journal of Differential Equations, vol. 2004, no. 108, 1C5, 2004.

[11] J. He and K. Wang, "The survival analysis for a population in a polluted environment," Nonlinear Analysis. Real World Applications, vol. 10, no. 3, pp. 1555-1571, 2009.

[12] S. Sinha, O. P. Misra, and J. Dhar, "Modelling a predator-prey system with infected prey in polluted environment," Applied Mathematical Modelling, vol. 34, no. 7, pp. 1861-1872, 2010.

[13] B. Liu, L. Chen, and Y. Zhang, "The effects of impulsive toxicant input on a population in a polluted environment," Journal of Biological Systems, vol. 11, no. 3, pp. 265-274, 2003.

[14] B. Liu, Z. Teng, and L. Chen, "The effects of impulsive toxicant input on two-species Lotka-Volterra competition system," International Journal of Information \& Systems Sciences, vol. 1, no. 2, pp. 208-220, 2005.

[15] X. Yang, Z. Jin, and Y. Xue, "Weak average persistence and extinction of a predator-prey system in a polluted environment with impulsive toxicant input," Chaos, Solitons \& Fractals, vol. 31, no. 3, pp. 726-735, 2007.

[16] F. Tao and B. Liu, "Dynamic behaviors of a single-species population model with birth pulses in a polluted environment," The Rocky Mountain Journal of Mathematics, vol. 38, no. 5, pp. 1663-1684, 2008.

[17] B. Liu and L. Zhang, "Dynamics of a two-species LotkaVolterra competition system in a polluted environment with pulse toxicant input," Applied Mathematics and Computation, vol. 214, no. 1, pp. 155-162, 2009.

[18] S. Cai, "A stage-structured single species model with pulse input in a polluted environment," Nonlinear Dynamics, vol. 57, no. 3 , pp. 375-382, 2009.

[19] J. Jiao and L. Chen, "Dynamical analysis of a chemostat model with delayed response in growth and pulse input in polluted environment," Journal of Mathematical Chemistry, vol. 46, no. 2, pp. 502-513, 2009.

[20] J. Jiao, W. Long, and L. Chen, "A single stage-structured population model with mature individuals in a polluted environment and pulse input of environmental toxin," Nonlinear Analysis. Real World Applications, vol. 10, no. 5, pp. 3073-3081, 2009.

[21] X. Meng, Z. Li, and J. J. Nieto, "Dynamic analysis of MichaelisMenten chemostat-type competition models with time delay and pulse in a polluted environment," Journal of Mathematical Chemistry, vol. 47, no. 1, pp. 123-144, 2010.

[22] J. Jiao, K. Ye, and L. Chen, "Dynamical analysis of a fivedimensioned chemostat model with impulsive diffusion and pulse input environmental toxicant," Chaos, Solitons \& Fractals, vol. 44 , no. $1-3$, pp. 17-27, 2011.

[23] J. Jiao, S. Cai, and L. Chen, "Dynamics of the genic mutational rate on a population system with birth pulse and impulsive input toxins in polluted environment," Journal of Applied Mathematics and Computing, vol. 40, no. 1-2, pp. 445-457, 2012.

[24] E. L. Johnston and M. J. Keough, "Field assessment of effects of timing and frequency of copper pulses on settlement of sessile marine invertebrates," Marine Biology, vol. 137, no. 5-6, pp. 10171029, 2000.

[25] R. M. May, Stability and Complexity in Model Ecosystems, Princeton University Press, 2001.
[26] Y. Kuang, Delay Differential Equations with Applications in Population Dynamics, Academic Press, Boston, Mass, USA, 1993.

[27] J. R. Beddington and R. M. May, "Harvesting natural populations in a randomly fluctuating environment," Science, vol. 197, no. 4302, pp. 463-465, 1977.

[28] R. Rudnicki and K. Pichór, "Influence of stochastic perturbation on prey-predator systems," Mathematical Biosciences, vol. 206, no. 1, pp. 108-119, 2007.

[29] L.-C. Hung, "Stochastic delay population systems," Applicable Analysis, vol. 88, no. 9, pp. 1303-1320, 2009.

[30] C. Zhu and G. Yin, "On hybrid competitive Lotka-Volterra ecosystems," Nonlinear Analysis. Theory, Methods \& Applications, vol. 71, no. 12, pp. el370-e1379, 2009.

[31] M. Liu and K. Wang, "Persistence and extinction in stochastic non-autonomous logistic systems," Journal of Mathematical Analysis and Applications, vol. 375, no. 2, pp. 443-457, 2011.

[32] M. Liu and K. Wang, "Stationary distribution, ergodicity and extinction of a stochastic generalized logistic system," Applied Mathematics Letters, vol. 25, no. 11, pp. 1980-1985, 2012.

[33] M. Liu, K. Wang, and Q. Wu, "Survival analysis of stochastic competitive models in a polluted environment and stochastic competitive exclusion principle," Bulletin of Mathematical Biology, vol. 73, no. 9, pp. 1969-2012, 2011.

[34] M. Liu and K. Wang, "Persistence and extinction of a singlespecies population system in a polluted environment with random perturbations and impulsive toxicant input," Chaos Solitons Fractals, vol. 45, pp. 1541-1550, 2012.

[35] M. Liu, H. Qiu, and K. Wang, "A remark on a stochastic predator-prey system with time delays," Applied Mathematics Letters, vol. 26, no. 3, pp. 318-323, 2013.

[36] M. Liu and K. Wang, "Population dynamical behavior of LotkaVolterra cooperative systems with random perturbations," Discrete and Continuous Dynamical Systems A, vol. 33, no. 6, pp. 2495-2522, 2013.

[37] M. Liu and K. Wang, "Dynamics of a Leslie-Gower Holling-type II predator-prey system with Lévy jumps," Nonlinear Analysis. Theory, Methods \& Applications, vol. 85, pp. 204-213, 2013.

[38] M. Liu and K. Wang, "Analysis of a stochastic autonomous mutualism model," Journal of Mathematical Analysis and Applications, vol. 402, no. 1, pp. 392-403, 2013.

[39] M. Liu and K. Wang, "Stochastic Lotka-Volterra systems with Lévy noise," Journal of Mathematical Analysis and Applications, vol. 410, pp. 750-763, 2014.

[40] N. Ikeda and S. Watanabe, "A comparison theorem for solutions of stochastic differential equations and its applications," Osaka Journal of Mathematics, vol. 14, no. 3, pp. 619-633, 1977.

[41] P. E. Kloeden and T. Shardlow, "The Milstein scheme for stochastic delay differential equations without using anticipative calculus," Stochastic Analysis and Applications, vol. 30, no. 2, pp. 181-202, 2012. 


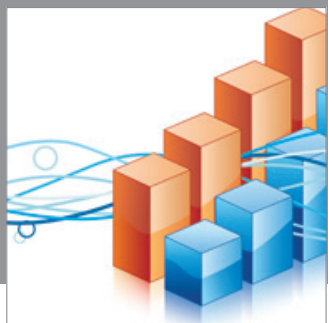

Advances in

Operations Research

mansans

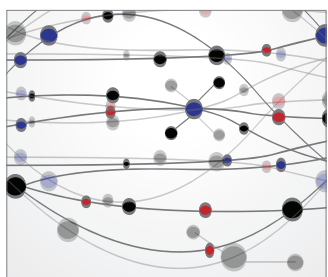

The Scientific World Journal
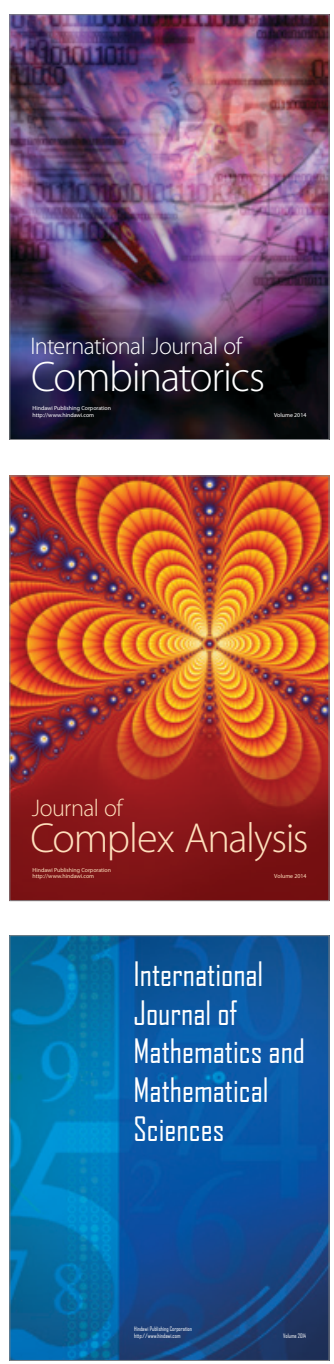
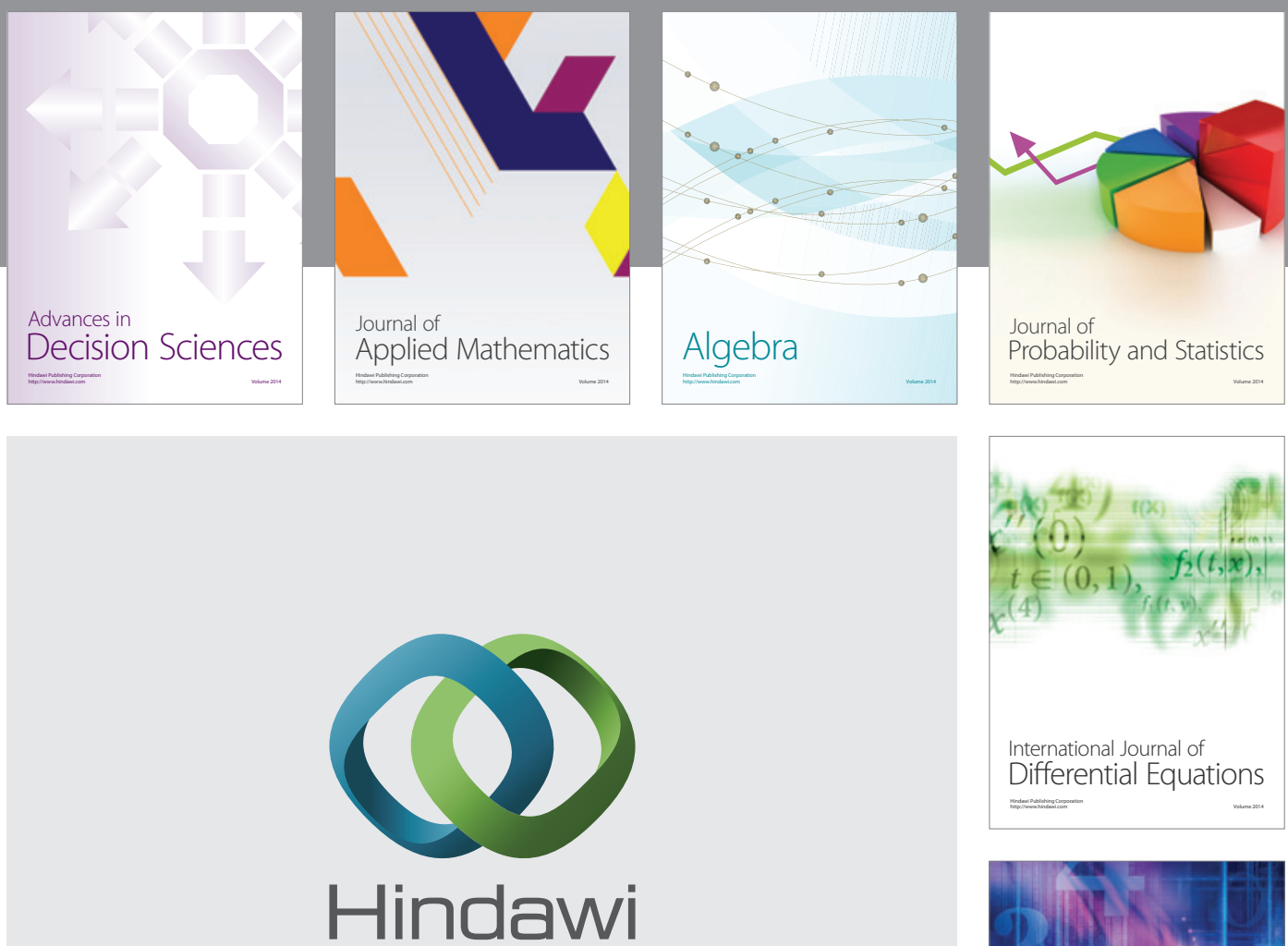

Submit your manuscripts at http://www.hindawi.com
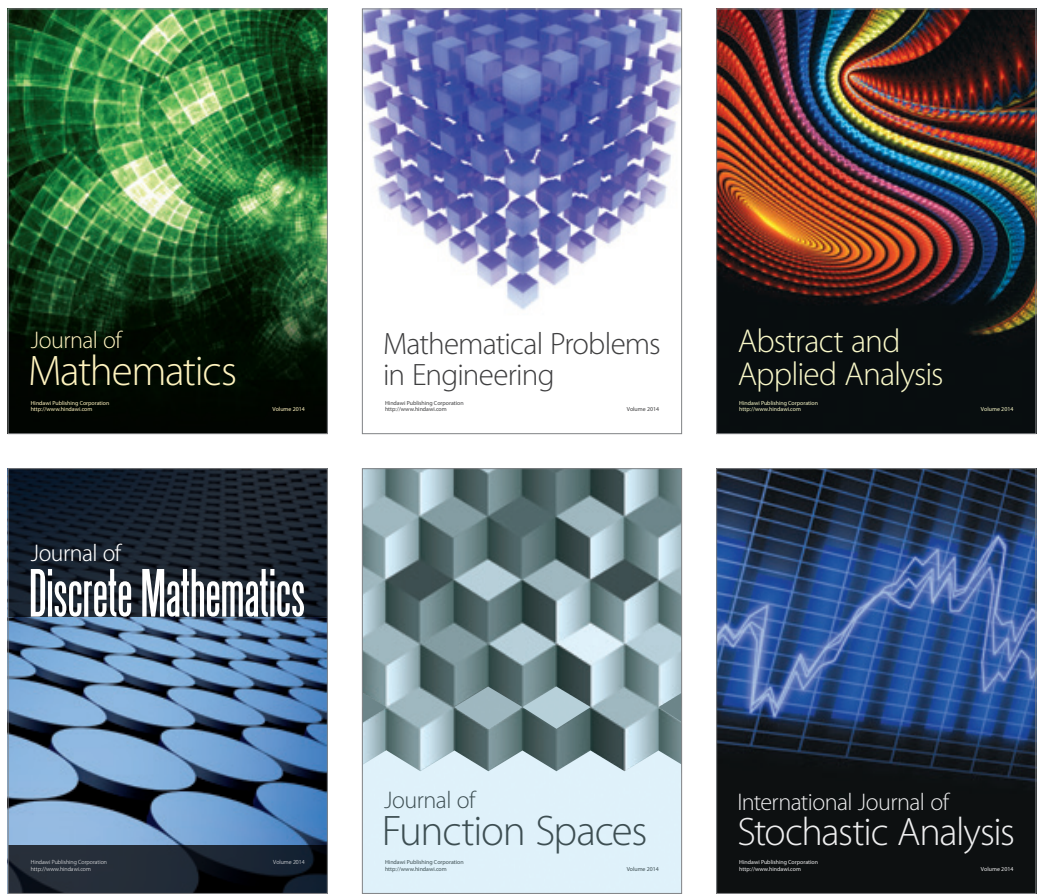

Journal of

Function Spaces

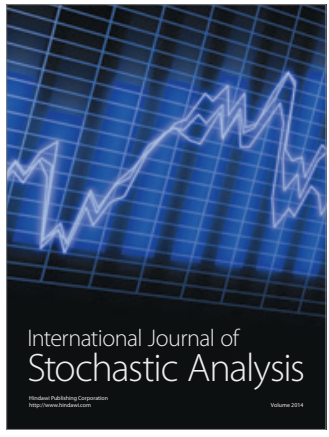

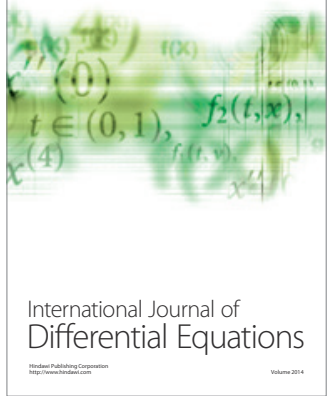
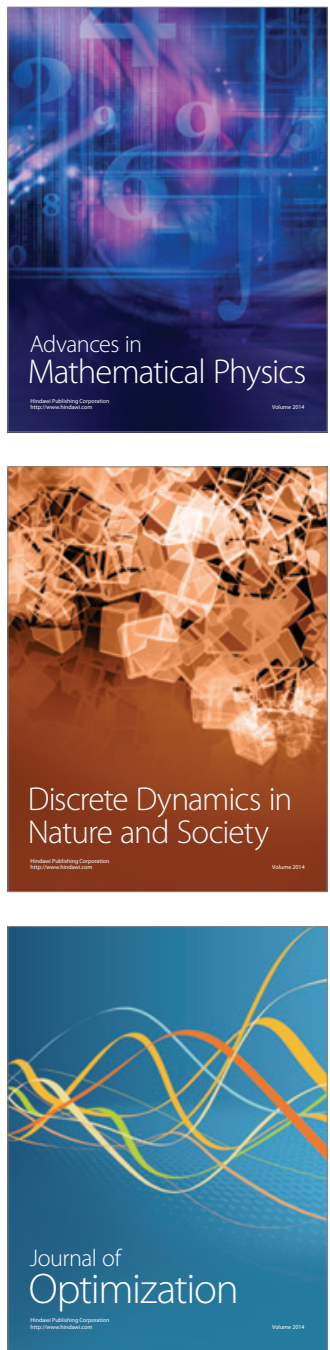